\title{
Developing a care pathway for advance decisions and powers of attorney: qualitative study
}

\author{
Jonathan I. Bisson, Victoria Hampton, Anne Rosser and Søren Holm
}

\section{Background}

The Mental Capacity Act for England and Wales empowers individuals to plan ahead for when they may lack capacity.

\section{Aims \\ To develop a care pathway for advance decisions and powers of attorney using Huntington's disease as an exemplar.}

\section{Method}

Qualitative study using in-depth individual interviews with service users and carers, and focus groups with professionals. Inductive qualitative analysis was used to develop themes to construct a care pathway that was then piloted and further evaluated to achieve a final pathway.

\section{Results}

A care pathway was developed that incorporated an early introduction through a formal education session and a minimum of two sessions separated by at least 2 weeks before advance decision completion. Optimal delivery of this intervention requires significant clinical and administrative commitment.

\section{Conclusions}

We have developed a simple, easy-to-follow care pathway that was acceptable to users and providers.

\section{Declaration of interest}

None. Funding detailed in Acknowledgements.
The Mental Capacity Act for England and Wales ${ }^{1}$ was implemented in October 2007. This legislation aims to empower individuals who may lose capacity and enable them to plan ahead by making an advance decision. Advance decisions have the potential to extend autonomy and provide clinicians and relatives with reassurance that management is consistent with what the individual would desire. ${ }^{2}$ They are likely to be particularly helpful to individuals with conditions that follow a partially predictable course such as most neurodegenerative conditions including most forms of dementia and many other life-shortening conditions. ${ }^{3}$

There is a lack of information regarding how best to approach advance decisions and powers of attorney with individuals who have or are likely to develop conditions that will result in loss of capacity. We therefore decided to use a qualitative method to develop a care pathway for advance decisions and powers of attorney using Huntington's disease as an exemplar. Most individuals with this disease will, at some stage, lose capacity to determine their ongoing care. Timely consideration of advance decisions seems particularly possible as most individuals with Huntington's disease are diagnosed before they lose capacity and, increasingly, individuals at risk elect to undergo predictive testing and are aware that they carry the gene before they develop the disease.

We aimed to address the following issues: when should advance decisions and lasting power of attorney be discussed; how should information regarding advance decisions and lasting power of attorney be delivered and by whom; how should capacity to execute an advance decision or lasting power of attorney be determined; and can a care pathway that is acceptable to service users and clinicians be developed.

\section{Method}

A qualitative approach was adopted based on Medical Research Council guidance. ${ }^{4}$ A prototype care pathway was developed through an initial modelling phase which was then piloted and evaluated. This led to a second prototype which was piloted and evaluated leading to the final care pathway.

\section{Participants}

\section{Modelling phase}

Purposive sampling was used to maximise the likelihood of obtaining a complete range of views. Individuals were invited to join a stakeholder group to contribute to the pathway development. The stakeholders included two individuals with symptoms of Huntington's disease, one carer, one asymptomatic individual who had the altered Huntington's disease gene, five clinicians working with individuals with symptoms of the disease, a lawyer with expertise in this area, a medical ethicist and two advisors employed by the Huntington's Disease Association (HDA). Theoretical sampling was used to ensure involvement of females and males, individuals of different ages and experience with different stages of Huntington's disease.

\section{Pilot phases}

During the pilot phases purposive sampling and the 'snowballing' technique, whereby those already approached were asked to identify other individuals who might be approached, were used. Participants were aged 18 or over, able to provide informed consent and in active contact with the South Wales Huntington's Disease Service. Six individuals with symptoms of the disease, nine carers/relatives, and four asymptomatic individuals with the altered Huntington's disease gene were included. Recruitment ceased when saturation of themes was achieved.

\section{Data collection}

\section{In-depth interviews}

In-depth interviews of up to $2 \mathrm{~h}$ conducted by J.I.B. were used to generate data from stakeholders who were service users or carers. In-depth interviews conducted by V.H. were used to generate data from individuals involved in the pilot phases.

\section{Focus groups}

Four focus groups facilitated by J.I.B. of $1-2 \mathrm{~h}$ with group sizes of between four and eight people were used to generate data from the 
other stakeholders. Two were conducted in the modelling phase and one after each pilot phase. Group interaction was encouraged and individuals were asked to clarify why they thought as they did to maximise data generation.

\section{Topic guide}

A topic guide was used for the in-depth interviews and focus groups that covered: information to be considered; presentation of information; methods of delivery; timing issues; individuals to be involved in the process; methods of communication; method of assessment of capacity; ${ }^{5,6}$ and form of documentation. Individuals interviewed after pilot phase participation were asked to consider their experience and views of the process.

\section{Analysis}

The in-depth interviews and focus groups were audio-taped and transcribed. Manuscripts were imported into QSR NVivo 7 (QSR International Pty Ltd, 2006) after participant approval. Relevant themes were identified using grounded theory in which data collection, analysis and systematic efforts to check and refine developing themes occurred concurrently. ${ }^{7}$ The emerging themes were tested for validity using a variety of recognised techniques ${ }^{8}$ including discussion of emerging themes; triangulation to compare the results from different sources; participant review of emerging themes; exploration of the respondents' underlying reasoning; and elements within the data that appeared to contradict the emerging themes ('deviant case analysis').

\section{Results}

\section{Modelling phase}

The main themes that emerged during the modelling phase could be grouped into five categories. These are described below and supported by the quotations of participants in Appendix 1 .

\section{Information to be considered and method of delivery}

Some confusion was apparent among service users regarding what advance decisions and powers of attorney are, not least the difference between advance decisions and euthanasia. Easy-tofollow, consistent verbal and written information was desired. Information specific to Huntington's disease was considered vital, especially regarding percutaneous endoscopic gastrostomy feeding and choices about location of care to guide future decisions. Participants felt that advance decisions would be optimally introduced through offering a leaflet at a clinic appointment with a brief verbal explanation. An individual could then choose to undergo further verbal education backed up by more detailed written information.

\section{Location and individuals involved}

The predominant opinion was that the location is an individual decision with some preferring their home and others a clinical setting. Having an established therapeutic relationship with an expert in Huntington's disease who facilitated the process emerged as a dominant theme. Personal qualities such as being approachable, caring and sensitive with good communication skills were felt to be important. Participants also recommended the additional offer of home visits by an HDA advisor.

\section{Timing and duration of process}

Professionals were reluctant to approach service users too early, particularly asymptomatic individuals with the altered Huntington's disease gene, for fear of causing distress. In contrast, service users had a more positive attitude towards early introduction of advance decisions in order to increase autonomy. A consensus was reached that the duration should be flexible allowing for as many sessions required to reach a decision. It was also considered important to have a minimum 2-week 'cool off' period between an initial meeting and advance decision completion.

\section{Assessment of capacity}

Service users and carers felt that capacity assessment was the responsibility of professionals but it should adhere to legal requirements. Professionals considered that the assessment should be decision-specific and adhere to the four levels of capacity stated in the Mental Capacity Act 2005. Tests such as the MacArthur Competence Assessment Tool for Treatment (MacCAT-T) ${ }^{9}$ may provide additional information to facilitate the assessment but should not be relied on. It was not felt vital for the capacity assessor to be from a specific discipline but it should be acknowledged that many professionals are reluctant to assess capacity and would require training to feel confident to do so.

\section{Form of documentation}

Participants recommended a single, short, easy-to-follow advance decision form with space for personal statements and wishes. The main issues that people believed should be on the form were: lifesaving treatments, percutaneous endoscopic gastrostomy feeding, location of future care, capacity assessment, witness details and a distribution list. A summary sheet for patient files, and checklists for education, completion and review were considered important.

As a result of the modelling phase, a prototype care pathway was developed along with a set of information leaflets and an advance decision document.

\section{Pilot phases}

During the first pilot phase, two individuals with Huntington's disease and two asymptomatic individuals with the altered Huntington's disease gene completed an advance decision. Two individuals with the disease decided not to complete an advance decision after the initial discussion. During the second pilot phase, one individual with Huntington's disease and one asymptomatic individual with the altered Huntington's disease gene completed an advance decision. One individual with the disease and one asymptomatic individual with the altered disease gene decided not to after the initial discussion.

Involvement in the care pathway was a positive experience for the majority in both pilot phases. One individual with Huntington's disease and their carer described feeling upset for a few days as a result of discussing end-of-life decisions that resolved without the need to seek external help. They continued to consider the care pathway important.

Those who did not complete an advance decision acknowledged the need for end-of-life issues to be raised to enable choice. For those who did complete an advance decision they found the process empowering and described achieving peace of mind because they were able to make decisions about a future which would otherwise be completely out of their control. Other benefits expressed included taking pressure off children, the importance of choices and gaining more information. 
The themes that emerged are described below and supported by Appendices 2 and 3 .

\section{Information to be considered and method of delivery}

General written information on wills and the Mental Capacity Act was requested along with more Huntington's disease-focused information on percutaneous endoscopic gastrostomy feeding. The power of attorney information was felt to be too detailed and a single booklet containing all the information provided recommended.

\section{Location and individuals involved}

Participants felt that given limited resources and clinician availability the formal meetings should take place in a clinical setting but that all individuals should be offered a home visit from an HDA advisor. One participant lived some distance from the hospital and requested an initial telephone interview. Both the participant and the facilitator were satisfied with the information delivered and interaction that occurred.

\section{Timing and duration of process}

A strong theme emerged that the earlier discussions regarding advance decisions are introduced the better, subject to checking personal circumstances and support, to allow consideration of them before individuals develop symptoms or their symptoms worsen. The increased difficulty in determining capacity of more symptomatic individuals was an additional argument for early introduction.

\section{Form of documentation}

The need to fully consider issues around impaired quality of life when making decisions was the major theme. It was also felt that the advance decision form should state whether it was Huntington's disease-specific or whether it applied whatever the cause of incapacity. Participants suggested adding statements concerning organ donation and whether independent legal advice had been received.

\section{Care pathways and documentation}

The emerging themes from the first pilot allowed the development of the second care pathway (Figs. 1, 2 and 3). A key difference from the original one was the earlier routine introduction to everyone including asymptomatic individuals with the altered Huntington's disease gene. A statement regarding quality of life was added to the advance decision documentation to help individuals who wanted to indicate when they would consider their quality of life so irreversibly impaired that active treatments should no longer be given. The results of the second pilot phase and evaluation led to some minor changes to the documentation but not the care pathway.

Stage 1 of the care pathway (Fig. 1) shows the introduction of end-of-life issues (advance decisions and powers of attorney) to individuals when they attend either pre-symptomatic or symptom management clinics by a professional with whom they have already formed a therapeutic relationship. The therapeutic relationship allows the professional to judge whether it is an appropriate time to raise this issue. There is an emphasis on end-of-life issues being a part of routine clinical education, not least to minimise feelings of it being talked about because of the severity of disease. The HDA leaflet Huntington's Disease and the Law is given, as it provides straightforward definitions of terms. ${ }^{10}$

Stage 2 outlines the education process and capacity assessment (Fig. 2). The duration of this stage is variable with a minimum of two sessions separated by a 2-week 'cool off' period for further deliberation and discussion. During the education, all points on the

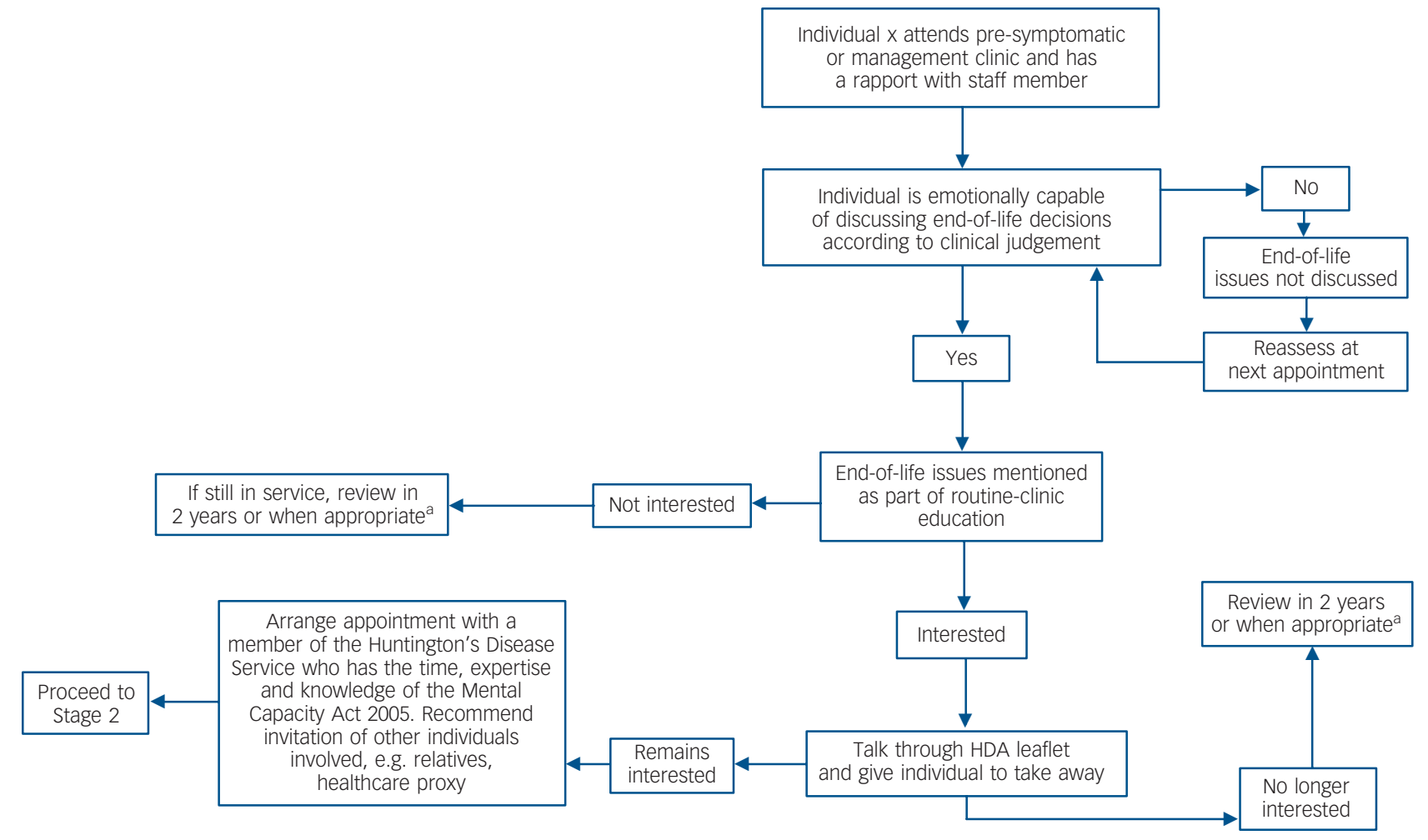

Fig. 1 Stage 1 shows the initial introduction of advance decision and powers of attorney to individuals in the clinic. 


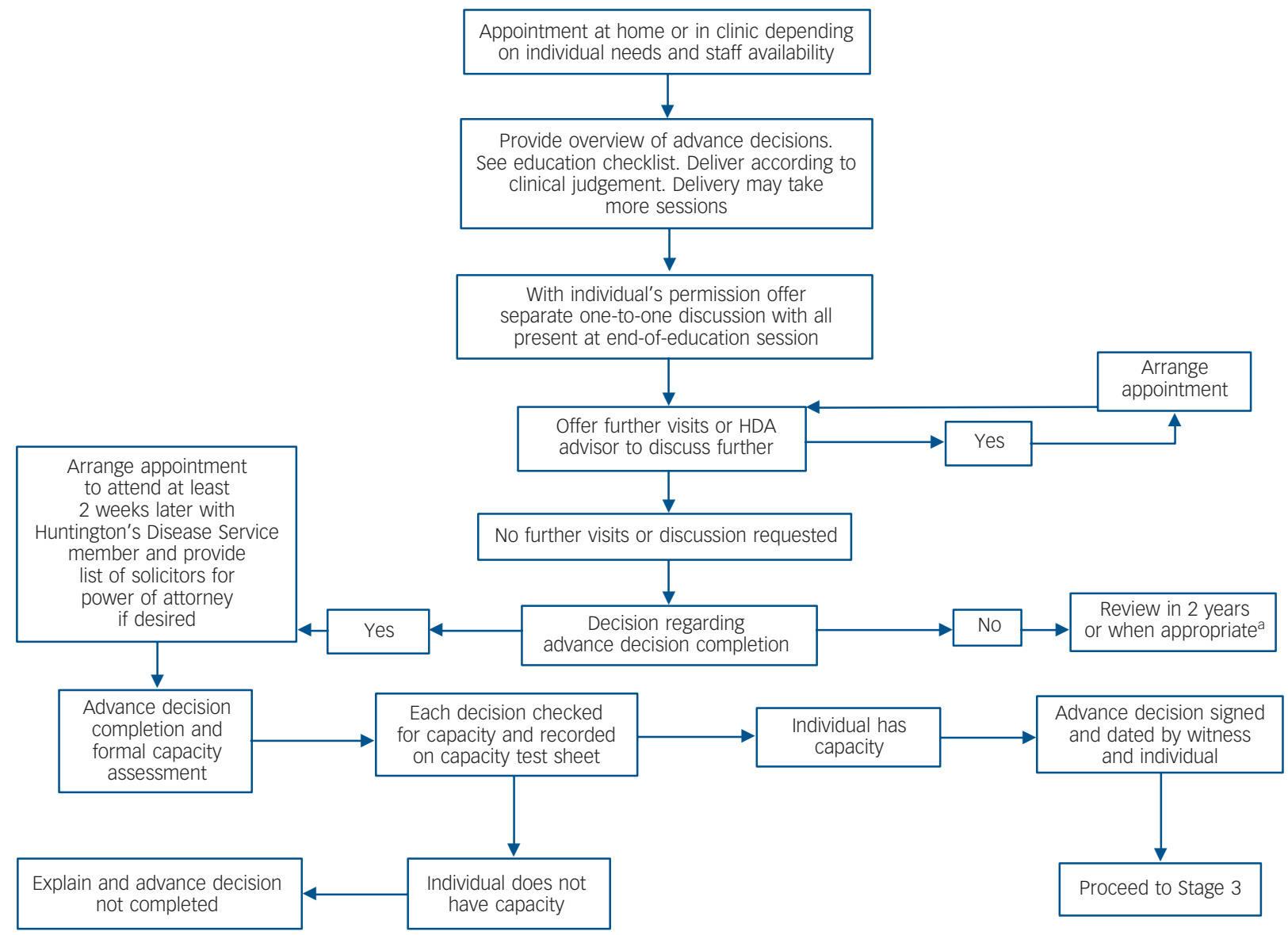

\section{Fig. 2 Stage 2 shows the education and capacity assessment process.}

HDA, Huntington's Disease Association.

a. 'When appropriate' refers to clinical judgement. An appropriate time will be when symptoms are increasing and individual is at significant risk of losing capacity in less than 2 years.

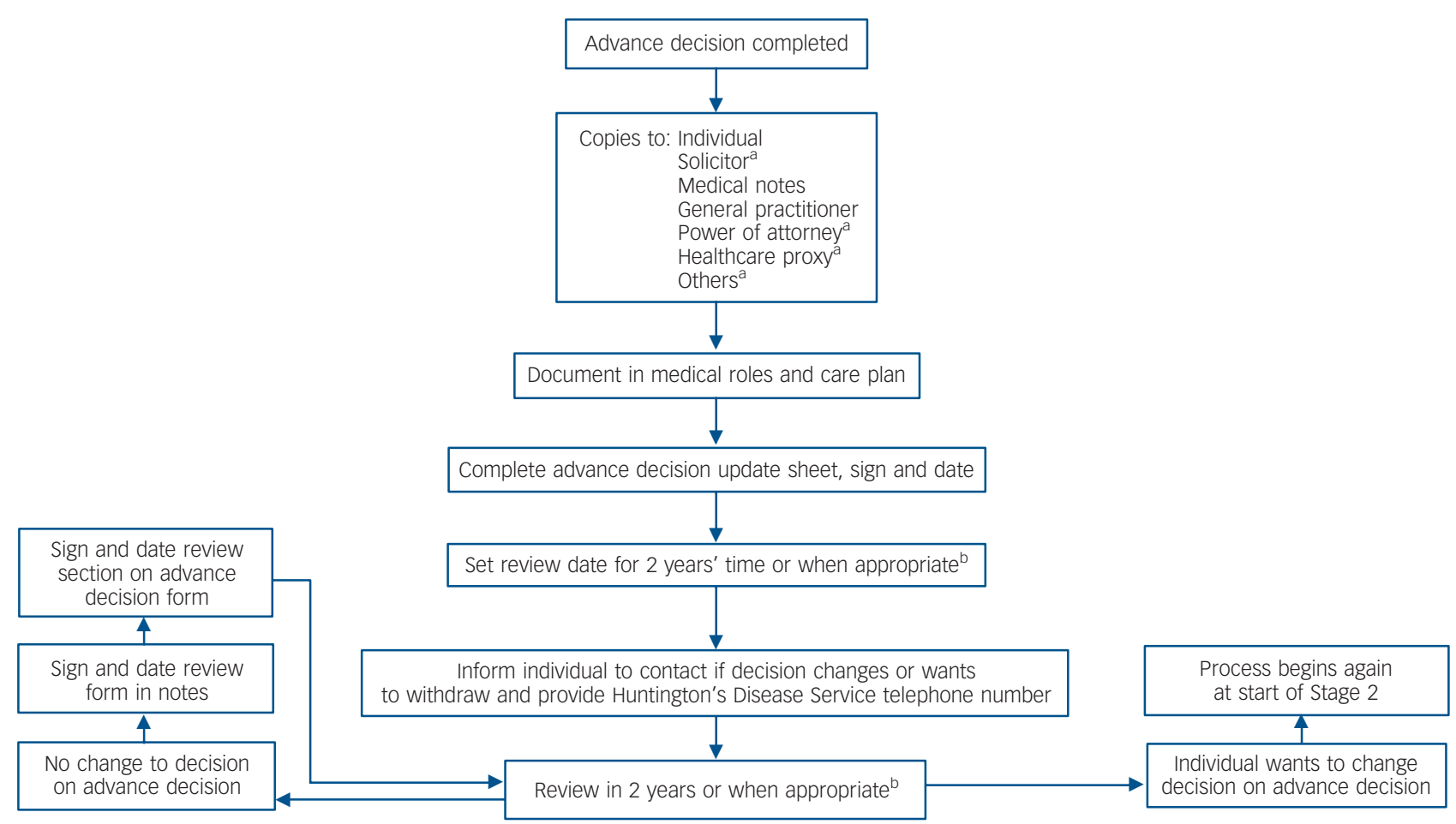

Fig. 3 Stage 3 shows the process after completion demonstrating the administrative commitment. 
checklist must be covered and all information provided before a decision is made. This may take several visits and a visit(s) from an HDA advisor. When education is complete and a decision to develop an advance decision formed the decision-specific capacity assessment can take place. Stage 3 details the completion process (Fig. 3). Copies are sent to individuals or places requested by the decision maker. Medical notes and update sheets are then completed and a review date set.

\section{Discussion}

\section{Principal findings}

This study produced a care pathway for the introduction and completion of advance decisions and powers of attorney. Individual choice and empowerment are emphasised. Optimal delivery requires significant clinical and administrative commitment.

\section{Strengths and weaknesses}

Robust qualitative methodology involving service users, carers and experts from different backgrounds was employed using Huntington's disease as an exemplar for many other conditions. The pathway was piloted and refined demonstrating its potential to be implemented into a clinical service. The study involved a relatively small but usual number of participants for a qualitative study. Caution is required in generalising the results but we consider them likely to be broadly representative because of the strong methodologies including the continuity of recruitment until there was saturation of themes at each stage of the process.

There is an absence of research concerning the development of care pathways regarding advance decisions and powers of attorney. The existing literature provides individual or committee recommendations that have not been developed through research.

\section{Clinical implications}

With the full implementation of the Mental Capacity Act 2005, ${ }^{1}$ advance decisions will be of increasing importance and need to be part of the clinical process. This study offers guidance for a wide range of chronic disease management services. Incorporating the pathway into routine service provision would be likely to raise awareness in both staff and service users and increase confidence in making advance decisions.

There are resource and staff implications that result from such a process. The average time of education sessions was an hour and for capacity assessment and decision completion another hour. Continued review at two-yearly interviews would also lead to lengthening of appointments. A $2 \mathrm{~h}+$ per person clinician commitment is likely to be very significant to many services without accounting for the additional administration required.

Introducing advance decisions may result in adverse emotional consequences in some individuals. Symptomatic individuals appeared to be more at risk in this study although an emotional reaction could occur in anyone. It is, therefore, strongly recommended that clinicians are aware of this. Early introduction is likely to be most beneficial but advance decisions should be raised with any individual with capacity who is affected by a dementia, other neurodegenerative condition or lifeshortening condition, or who has the adverse result for the altered gene in the case of Huntington's disease.

Unfortunately for many people, clinical management decisions are only considered when they no longer have capacity. One study found that only $20 \%$ of individuals with early dementia were competent to complete an advance decision. ${ }^{11}$ This often results in them never being considered. Other countries have brought in legislation to counter this. In the USA, providers of care are required to advise patients of their right to accept or refuse medical care and to execute an advance decision in the event of incapacity. ${ }^{12}$ Several commentators have expressed major ethical reservations particularly about the meaning, reliability, durability and portability of advance decisions. ${ }^{13}$

Some researchers have described a process of advance care planning in which patients and carers discuss decisions together before capacity is lost. ${ }^{14}$ Other key issues include whether an advance decision made with capacity to refuse treatment should always take precedence over an acceptance of treatment when capacity is diminished; what happens when personality alters as a result of disease resulting in different values; and the impact of response shift in which individuals adapt to circumstances that in the advance decision would trigger the denial of treatment. These can be at least partially ameliorated by appointing a person with lasting powers of attorney to interpret the advance decision, or to make decisions on situations the advance decision does not cover.

\section{Future research}

Work is now required to develop an implementation package of the findings of this study and to monitor the usefulness of the care pathway when delivered as part of routine clinical care. This would include production of a booklet containing the educational materials and a training package for professionals enabling them to feel confident and competent to deliver the advance decision pathway and to assess decisional capacity.

\section{Jonathan I. Bisson, DM, FRCPsych, Victoria Hampton, BSC, Department \\ of Psychological Medicine, Cardiff University, University Hospital of Wales: \\ Anne Rosser, PhD, FRCP, Departments of Neurology and Medical Genetics, Cardiff \\ University, University Hospital of Wales; Søren Holm, MD, PhD, Cardiff Law School, Cardiff University, Museum Avenue, Cardiff, UK \\ Correspondence: Jonathan I. Bisson, Department of Psychological Medicine, Cardiff University, Monmouth House, University Hospital of Wales, Heath Park, Cardiff CF14 4XN, UK. Email: bissonji@cf.ac.uk}

First received 13 Dec 2007, final revision 10 Jun 2008, accepted 21 Aug 2008

\section{Acknowledgements}

We thank all participants in the study and the Welsh Office for Research and Development for funding it.

\section{Appendix 1}

\section{Quotations associated with the modelling phase}

Information to be considered and method of delivery

'I guess there's a crossover with euthanasia and people knowing the difference between them. I think it might be useful to tell people about the difference between them because I think there might be some confusion.' A - service user

'Maybe give people a simplified leaflet that's just a couple of pages and then any more details could be discussed at a specific visit with them.' F - professional

\section{Location and individuals involved}

'I think that it depends on the individual abilities and physical state and who they have supporting them so that they feel more comfortable. I mean, if you're visiting someone in their home then that's fine, if they're used to coming and seeing someone in clinic then that's probably okay. I think it would have to be an individual decision depending on the person.' $\mathrm{K}$ - professional 
'Well, it has to be somebody who is an expert in the disease and that could be of any discipline but someone who is very versed in the condition.' $\mathrm{K}$ - professional

\section{Timing and duration of process}

'In order for the individual to have the most control, the discussion should take place earlier. The earlier the better really.' $\mathrm{H}$ - professional

'I think if I had symptoms, then I'd be panicking to rush this thing through.' B - service user

\section{Assessment of capacity}

'Clinical assessment of capacity according to the criteria is the key thing and that can be done by anyone, but people with more psychiatric consequences may need someone with more expertise.' $\mathrm{K}$ - professional

\section{Form of documentation}

'I would say it should be a standardised document and additional information could be filled in by speaking to the person. I'd say that was the easiest way to do it.' I - professional

(Letters refer to identity of participant quoted)

\section{Appendix 2}

\section{Quotations associated with the first pilot phase}

Information to be considered and method of delivery

'The Huntington's Disease Association leaflet was actually the best one of all. It gave a lot of information but it's not too in-depth either.' $\mathrm{V}$ - service user

Location and individuals involved

'I think it would be comfortable if it was made less like a hospital appointment. It's not a hospital appointment. It is something very important to us. $\mathrm{S}$ - carer

'It helped that we know him. I wouldn't have wanted someone I didn't know. It made it easier. We have a rapport with him.' Q - carer

\section{Timing and duration of process}

'Even though I went away from here thinking, "I don't really need this" did actually find it useful [2-week 'cool off' period]. It made me think. The two visits were needed.' $\mathrm{B}$ - service user

\section{Form of documentation}

'We weren't sure about the options on life-threatening conditions. I spoke to my family about it and we were saying about quality of life. Each of us had a different opinion on what a decent quality of life is. What we had to do in the notes was write there what I class as a decent quality of life. That's what this is really about, quality of life.' $V$ - service user

\section{Opinions}

'It's been exhilarating for me because it's put my life in order.' B - service user

'I was okay when I was in the room but then when I went away to think about it that's when it hit me and I thought about what is to come. I know I've been through it before but it's the reality of it.' Q - carer

'It has been a really positive experience for both of us. There are so many things to worry about and now that this is done we have one less thing to worry about.' S - carer

(Letters refer to identity of participant quoted)

\section{Appendix 3}

\section{Quotations associated with the second pilot phase}

Information to be considered and method of delivery

'I think it would be more user-friendly if we had an actual book.' 0 professional

'The information in the [HDA] leaflet wasn't patronising. It was straight to the point and it was easy to understand.' $\mathrm{C} 2$ - service user

'It was very good. It was good that the doctor played devil's advocate, you know, "are you sure you want this and do you realise that this will happen and that." ' D2 - carer

'[re telephone call] I thought it was good. I think he has a natural style of going over things as well and saying I understand that we confirm this and I think that removes any of the problems like a misunderstanding or things that you can't pick up from people's facial expressions . . . I would certainly see it as onerous to have to do lots of visits to do this, so being able to do things over the telephone is a huge advantage. ' $Y$ - service user

Location and individuals involved

'I did wonder why [he was a psychiatrist]. I suppose because you have to show that you are thinking rationally ... It wouldn't have made any difference if the genetics nurse had done it as far as I was concerned. She could have sat there and gone through it.' D2 - carer

'I think it helped me having someone that I know explain these things to me. It needs to be somebody who's caring when you talk to them. It's a major thing to think about really ... I think somebody who is an expert in Huntington's disease would probably be good. I don't think it has to be a psychiatrist.' $X$ - service user

\section{Timing and duration of process}

'I think maybe people might need a bit longer because it's a big decision and there's lots of things to consider and think of. So from my point of view 2 weeks wasn't enough . . . Maybe 4 weeks would be good.' $\mathrm{X}$ service use

'That was fine, didn't need anymore ... I had all that made up about the stuff beforehand so for me the two sessions were enough.' D2 - carer

\section{Assessment of capacity}

'I think it would be fair to say that was the toughest part of it . . . She was getting really worked up and we had to stop a bit. For her it was difficult. . . I think the assessment part was the difficult bit because the later on in the disease you are then the more difficult it is to know what they are saying.' B2 - carer

\section{Form of documentation}

'Quality of life is very important to be on the form ... It's good because it gives you room for what you want to put down, which is more important because it's quite personal what people consider quality of life and what they want to be treated for ... Everybody with Huntington's disease will want different things for themselves.' $\mathrm{X}$ - service user

'It was useful to have the sheet of paper with statements [about quality of life and possible options] because I'm a bit rubbish at that sort of thing.' D2 - carer

\section{Opinions}

'It's unfortunate that things like this hadn't been available for my mother and my grandmother, having seen them and all the family arguments that it has caused.' D2 -carer 
'For me it was probably better than for her. I know that nobody can interfere with what we've put down. It's all written down and everybody knows what the score is.' B2 - carer

'Good. It's what I wanted and it's done.' A2 - service user

(Letters refer to identity of participant quoted)

\section{References}

1 Department for Constitutional Affairs. Mental Capacity Act 2005 Code of Practice (2007 final edition). TSO (The Stationery Office), 2007.

2 Lancet. Advance directives [editorial]. Lancet 1992; 340: 1321-2.

3 Singer P. Disease-specific advance directives. Lancet 1994; 344: 594-6.

4 Medical Research Council. A Framework for Development and Evaluation of RCT's for Complex Interventions to Improve Health. Medical Research Council, 2000 (http://www.mrc.ac.uk/Utilities/Documentrecord/ index.htm?d=MRC003372)

5 Fazel S, Hope T, Jacoby R. Assessment of competence to complete advance directives: validation of a patient centred approach. BMJ 1999; 318: 493-7.
6 Raymont V, Bingley W, Buchanan A, David AS, Hayward P, Wessely S, et al. Prevalence of mental incapacity in medical inpatients and associated risk factors: cross-sectional study. Lancet 2004; 364: 1421-7.

7 Pope C, Mays N. Reaching the parts other methods cannot reach: an introduction to qualitative methods in health and health services research. BMJ 1995; 311: 42-5.

8 Mays N, Pope C. Assessing quality in qualitative research. BMJ 2000; 320 : 50-2.

9 Grisso T, Appelbaum PS, Hill-Fotouhi C. The MacCAT-T: a clinical tool to assess patients' capacities to make treatment decisions. Psychiatr Serv 1997; 48: 1415-9.

10 Huntington's Disease Association. Huntington's Disease and the Law. HDA 2006 (http://www.hda.org.uk/download/acrobat/hdafs012.pdf)

11 Fazel S, Hope T, Jacoby R. Dementia, intelligence and the competence to complete advance directives. Lancet 1999; 354: 48.

12 Ulrich L. The Patient Self-Determination Act. Meeting the Challenges in Patient Care. Georgetown University Press, 1999

13 Holm S. Autonomy, authenticity, or best interest: everyday decision-making and persons with dementia. Med Health Care Philos 2001; 4: 153-9.

14 Martin $D$, Thiel E, Singer P. A new model of advance care planning: observations from people with HIV. Arch Intern Med 1999; 159: 86-92.

\section{Poems by doctors}

\section{Knees}

\section{For Kate}

\section{Stephen Wilson}

Everywhere a wasteland of clapped out muscle, unhinged wheely-bins overflowing with trash;

if you scope the comings and goings, you'll find a murky dive,

where inarticulate jerks and low-lifes

meet in an overcrowded space -

a steamy room with loose bodies

locked in unusual positions,

caught on camera, moving slowly,

if at all. A tenor sax bends Mood Indigo

through its horn and behind the bar

a notice tells you not to ask for credit

as a refusal often offends. There are beds

out the back and the floor's wet, where bits

of ragged tissue have been left around since

something went, after a night on the tiles.

Who knows which gang's running the protection

racket in those crooked joints, nothing like the bee's.

Stephen Wilson's first collection of poems, Fluttering Hands, was published by Greenwich Exchange in April 2008. He was born in London in 1944, trained at the Royal Free Hospital and qualified as a doctor in 1968. His early experience of psychiatry was at Fulbourn Hospital, Cambridge. In the 1970's he moved to Oxford where he worked for many years at Littlemore and later as a consultant psychotherapist in the Warneford Hospital. He became a Fellow of the Royal College of Psychiatrists in 1985 and was formerly Honorary Senior Clinical Lecturer in the Department of Psychiatry, University of Oxford. During the period 1989-1994 he was an assistant editor for the British Journal of Psychiatry and founding editor of the BJP Review of Books. He retired from medical practice in April 2008.

His poems have appeared in the London Review of Books and other magazines and anthologies. He is also the author of The Bloomsbury Book of the Mind, Introducing the Freud Wars, Sigmund Freud (A Pocket Biography) and The Cradle of Violence: Essays on Psychiatry, Psychoanalysis and Literature. He has contributed numerous reviews and articles to academic journals and newspapers including Encounter, TLS, The Guardian, The Independent and the New York Times.

Another of Stephen Wilson's poems was published in the December 2008 issue of the Journal. Reprinted with the permission of Greenwich Exchange. 